\title{
Circadian Timing and Regularity of Physical Activity: A Novel Bioprocess to Prevent Devastating Modern Diseases
}

\section{Akbar Nikkhah*}

Chief Highly Distinguished Professor, Department of Animal Sciences, University of Zanjan, Iran

This article develops a novel global strategy to prevent and manage cancer and obesity and other major modern diseases by optimizing circadian timing and regularity of physical activity. Early morning and early evening are considered beneficial in optimizing endocrinology and metabolism. This optimization, particularly in early evening or late afternoon, has the great potential to minimize risks of obesity, diabetes and related cardiometabolic concerns.

\section{Innovative Discussion}

Circadian timing of human life has greatly influenced survivorship and resistance against a variety of environmental pathogens. The major life affairs including eating, resting, working, and exercising must accordingly possess their own optimal circadian timing that fits internal chronophysiological rhythms optimally. Recent discoveries have elaborated on the significance of circadian timing of eating on human physiology and health [1-5]. Optimal timing of eating in relation to exercise can also potentially and greatly improve gut health [6]. Despite the great needs of complementary research on the topic, very recently, global theories on the importance of matching the circadian rhythms of eating with those of physical activity and resting were reasonably substantiated [7-10].

For instance, evening and night food meals are considered very risky in terms of central adiposity, obesity and diabetes development. This suggests that should evening or night meals be taken, due to the increased likelihood of insulin resistance and glucose intolerance, fitting physical activity with adequate intensity and duration must be scheduled on a circadian and regular basis. As recently innovated, a regular daily exercise program that ensures adequately intense physical work has the potential to eradicate the factors contributing to cancer development [11-14].

Early morning is a time when the human body is rhythmically prepared to assimilate and metabolize energy-producing substrates of mainly glucose, amino acids and fatty acids. To elaborate on, this means that morning meals (i.e., timely breakfast and post-breakfast) do not usually impose any significant risk to metabolic health. This is because the human body has evolved to be most active during morning and day-time when physical work is in peak, and thus, the morning meals can be easily processed towards meeting energy requirements of brain and periphery notably muscles. This is, however, not the case in evening and overnight when the body is prepared to merely rest. As a result, substrate overload easily occur, thus increasing the risk of evening and nocturnal diabetes and accumulated weight gain. Based on this logic, pre-meal early morning and early evening exercise help the body increase its tolerance of substrate overload post-meal and to get gradually and effectively adapted to a rhythmic circadian alarm to minimize insulin resistance and obesity. However, it must be cared very seriously that early-evening or late afternoon exercise must not be followed by any large food meal to not exacerbate the glucose overload, which would otherwise provide no metabolic and health benefits over no exercise.

This article suggests that either of early morning and early evening (or late afternoon) physical activity can be greatly advantageous to various cells and organs. However, this article does not suggest that exercise must necessarily be conducted twice daily in both morning and evening times. It may be done so, but it is not essential. Since most people work during day, afternoon and evening exercise could be fairly refreshing, entertaining, extra energy-burning, and relaxing, when compared to morning exercise. This is more important when time for deep resting and sleep is limited in the hectic today's life. Future research needs to explore more science on the several key aspects of exercise timing and its relationship to oncogenesis and measures of cardiovascular health and quality of life.

\section{Implications}

This article described an innovative feasible strategy to prevent and manage major human diseases of mainly cancer, obesity, and cardiovascular issues through optimizing circadian timing of physical activity. Early morning and early evening/late afternoon intense exercise are considered beneficial towards optimal endocrinology and metabolism involving insulin work and obesity and related diseases prevention. Early evening is a special time for, at the very least, a brief intense exercise to relatively reduce nocturnal insulin resistance and improve energy tolerance.

\section{Acknowledgments}

Thanks to Iran's Ministry of Science Research and Technology, National Elite Foundation, and University of Zanjan for supporting the author's global initiatives and programs of optimizing science edification and dissemination.

\section{References}

1. Nikkhah A (2012) Time of Feeding an Evolutionary Science. Lap Lambert Publishing, GmbH \& Co. KG, Germany.

2. Nikkhah A (2015) Intake Circadian Physiology: An Overlooked Public Health Concern. Endocrinol Metab Synd 4: 153.

3. Nikkhah A (2015) Avoid Large Night Meals to Stay Fit. J Obes Weight Loss Ther 4: e115.

4. Nikkhah A (2014) Disease Closure through Opening Novel Chrono-Sciences: Bioprocessing of Intermediary Metabolism. J Bioprocess Biotech 5: e118.

5. Nikkhah A (2014) When to Eat to Beat Obesity and Diabetes? J Diabetes Metab 5: e115.

6. Nikkhah A (2015) Living Gut Health Improvement through Time-Managing

*Corresponding author: Akbar Nikkhah, Chief Highly Distinguished Professor Department of Animal Sciences, Faculty of Agricultural Sciences, University of Zanjan, Zanjan, Iran, National Elite Foundation, Tehran, Iran, Tel: +98-24-350328-01; Fax: +98-24-350-332-02; E-mail: nikkhah@znu.ac.ir

Received May 17, 2015; Accepted May 20, 2015; Published May 25, 2015

Citation: Nikkhah A (2015) Circadian Timing and Regularity of Physical Activity: A Novel Bioprocess to Prevent Devastating Modern Diseases. J Bioprocess Biotech 5: e131 doi:10.4172/2155-9821.1000e131

Copyright: @ 2015 Nikkhah A. This is an open-access article distributed under the terms of the Creative Commons Attribution License, which permits unrestricted use, distribution, and reproduction in any medium, provided the original author and source are credited. 
Nutrient Assimilation: An Evolutionary Probiotic. J Prob Health 3: e114

7. Nikkhah A (2015) Creating Healthy Endocrinological Patterns through Regular Daily Exercise: A Global Guideline. Endocrinol Met Synd.

8. Nikkhah A (2015) Nature as an Ideal Rhythm Model for Optimal Cardiovascular Physiology and Health. Int J Diabetol Vasc Dis Res 3: 1-2

9. Nikkhah A (2015) Secure Weight Management via Fitting Circadian Patterns of Physical Activity, Resting and Eating. Adv Obes Weight Manag Cont 2: 023.

10. Nikkhah A (2015) Effective Weight Management in Periparturient Women through Optimizing Eating Timing: A Novel Global Approach. Adv Obes Weight Manag Cont 2: 018.
11. Nikkhah A (2015) Circadian Timing of Hunger to Avert Obesity: An Environmental Evolutionary Science. J J Environ Sci 1: 005.

12. Nikkhah A (2015) Circadian Fitting of Exercise and Eating Patterns: The Secret of Healthy Life. J Bioprocess Biotech 5: e129.

13. Nikkhah A (2015) Harmonizing Eating and Exercise Circadian Rhythms for Optimal Glucose-Insulin and Vascular Physiology. Int J Diabetol Vasc Dis Res 3: 87-88.

14. Nikkhah A (2015) Breast Safety Progress through Exercise-Driven Lactation: A Pragmatic Bioprocess to Prevent Cancer. J Bioprocess Biotech 5: e127. 